YITP-SB-00-61

\title{
New instantons in the double-well potential
}

\author{
Stefan Vandoren and Peter van Nieuwenhuizen \\ C.N. Yang Institute for Theoretical Physics \\ State University of New York at Stony Brook \\ NY 11794-3840, Stony Brook, USA \\ vandoren, vannieu@insti.physics.sunysb.edu
}

\begin{abstract}
A new solution of the Euclidean equations of motion is found for the quantummechanical double-well potential with a four-fermion term. It extends the usual kinkinstanton solution in which both the kink field and the fermionic fields contain a finite number of new terms which are polynomial in the fermionic collective coordinates. The solution has finite action, $S=-\frac{m^{3}}{3 \lambda}+\frac{9}{140} m g \epsilon^{i j k l} \xi_{i} \xi_{j} \xi_{k} \xi_{l}$, where $\xi_{i}, i=1, \ldots, 4$, are fermionic collective coordinates and $g, m$ and $\lambda$ are coupling constants. We explain why in general the instanton action can depend on collective coordinates.
\end{abstract}

\footnotetext{
${ }^{1}$ Work supported by NSF grant PHY-972 2101.
} 


\section{Introduction}

Instantons are finite action solutions to the Euclidean equations of motion in a given field theory, and describe tunnelling processes between different vacua. When the model possesses rigid symmetries, one can act with the transformation rules on a solution to generate new solutions, and so the instanton depends on a number of collective coordinates. The instanton action is typically inversely proportional to the coupling constant and does not depend on the collective coordinates generated by the rigid symmetries. Examples are the instanton in pure Yang-Mills theories [1], or the instanton in the quantum-mechanical double-well potential (see e.g. [2]).

In supersymmetric theories, or more generally in a theory containing fermions and bosons, the equations of motion become more complicated because the fermions can couple non-trivially to the bosonic fields. An obvious solution is to set all fermions to zero, and to take for the bosonic fields the usual instanton. When rigid supersymmetry is present, one can use the supersymmetry transformation rules to obtain other exact solutions of the equations of motion with the fermions non-zero and containing fermionic collective coordinates. Because this configuration is related to the purely bosonic one by supersymmetry, the instanton action is still the same and does not depend on the fermionic collective coordinates.

However, the most general solution to the equations of motion can often not be obtained by acting on the purely bosonic configuration with symmetries. As an example consider a chiral spinor, say in the adjoint representation of an $S U(N)$ gauge theory with $N \geq 3$, satisfying the Dirac equation in the presence of a single instanton. The number of solutions is given by $2 N$, so there are $2 N$ Grassmannian collective coordinates (GCC). Such a spinor field is present in $\mathcal{N}=1 S U(N)$ supersymmetric Yang-Mills theory. Using ordinary and conformal supersymmetry, one can generate four GCC, but the remaining solutions of the Dirac equation are not directly obtained by symmetryt. Nevertheless, because the fermions are just minimally coupled to the gauge fields, the instanton action is still the same as without the fermions (and hence independent of the GCC), because the fermion kinetic term vanishes upon using the Dirac equation.

The question we want to address in this paper is whether the instanton action can depend on collective coordinates which are not produced by any rigid symmetry. Contrary to what is commonly believed, we will show in an explicit example that the instanton action can indeed depend on the collective coordinates of the exact instanton solution. In the discussion we shall comment on whether this solution can be interpreted as an instanton, and whether it is related to other work in the literature. If we denote a collective coordinate by $\gamma$ and the fields by $\phi$, the

\footnotetext{
${ }^{2}$ However, these other fermionic solutions have also been given a geometrical interpretation, related to the orientation of the $S U(2)$ subgroup in $S U(N)$ [3].
} 
dependence of the action on $\gamma$ can be computed from

$$
\frac{\partial S}{\partial \gamma}=\left.\int \mathrm{d} x \frac{\partial S}{\partial \phi(x)}\right|_{\phi_{\mathrm{cl}}} \frac{\partial \phi_{\mathrm{cl}}}{\partial \gamma}
$$

with $\phi_{\mathrm{cl}}$ the exact instanton solution. It is then sometimes argued that because $\partial S / \partial \phi=0$ is the field equation, which is satisfied by $\phi_{\mathrm{cl}}$, the action cannot depend on $\gamma$. A closer inspection of (1.1) however shows that this argument is not correct. The reason is that to obtain the field equation, one has to do a partial integration. The flaw in the argument is then that one has neglected the surface term that appears when doing the partial integration: in the instanton background it can yield a non-zero and finite contribution. We will show this explicitly in a quantum-mechanical model.

In theories where the fermions are not minimally coupled, like the model we will consider in this paper, or when other fields are present which couple non-trivially, the equations of motion are much harder to solve. Another interesting example is the $\mathcal{N}=4$ supersymmetric Yang-Mills theory, which contains, besides the gauge fields, four Weyl spinors and six scalar fields, all in the adjoint representation. The equation of motion for the fermions is not the Dirac equation, but contains inhomogeneous terms due to the presence of Yukawa interactions. Again, the instanton solution of [目] with all scalars and fermions vanishing is an exact solution of this model, but one can also include the fermionic zero modes and try to obtain another exact solution. Even when the scalar fields have no vacuum expectation valuef, it is difficult to solve the equations of motion with all fermionic collective coordinates present. The strategy one follows is to solve the equations of motion iteratively in the number of GCC. Because of the Grassmannian nature, this is a finite procedure. For $\mathcal{N}=4$ super Yang-Mills theory, this procedure was initiated in [6], and continued in more detail in [7], to lowest order in the GCC. It was also shown that after one iteration the instanton action contains a term proportional to four GCC, see also [8]. But because of the complicated structure of the model (the number of iterations grows with the $N$ of $S U(N)$ ), an exact solution was not obtained. It is important to find the complete GCC dependence of the instanton action, since these terms are needed for computing correlation functions beyond the leading order in the coupling constant. Specifically, we have in mind the instanton calculations which are used to check the AdS/CFT correspondence in $\mathcal{N}=4$ super Yang-Mills theory. It is unknown whether a complete solution exists in this model, whether it has finite action, and whether the winding number is modified.

\footnotetext{
${ }^{3}$ If there is a vacuum expectation value for the scalars, one can use Derrick's theorem to prove there are no exact solutions to the equations of motion 泊. In such cases, one works with approximate solutions, called constrained instantons $[5]$. The instanton action then depends on the collective coordinates because the field equations are not satisfied.
} 
The purpose of this letter is to use a toy model where this iteration procedure can be demonstrated explicitly. It is a deformation of the instanton in a supersymmetric version of the quantummechanical model with a double-well potential. As we will see, the iteration will only take two steps, and hence our construction leads to a new instanton solution with non-vanishing fermions. We compute the instanton action, show that it is finite and contains terms proportional to four fermionic collective coordinates.

\section{The model}

We start by considering the following quantum mechanical model, formulated in Euclidean space,

$$
S_{\text {bos }}=-\frac{1}{2} \int \mathrm{d} \tau\left\{\dot{x}(\tau)^{2}+U^{2}(x)\right\},
$$

where the dot stands for the $\tau$-derivative, and the integration is over the infinite $\tau$-line. The potential has a double-well shape,

$$
\frac{1}{2} U^{2}=\frac{1}{4} \lambda\left(x^{2}-\frac{\mu^{2}}{\lambda}\right)^{2} .
$$

The field equation, $\ddot{x}-U U^{\prime}=0$, where the prime stands for the $x$ derivative, has the usual kink-instanton solution,

$$
x_{K}(\tau)=\frac{\mu}{\sqrt{\lambda}} \tanh \left[\frac{\mu}{\sqrt{2}}(\tau-X)\right],
$$

where $X$ is a collective coordinate associated with time translations. In fact, the instanton satisfies the first-order BPS equation $\dot{x}_{K}+U\left(x_{K}\right)=0$.

In the spectrum of fluctuations around the kink-instanton, one finds a zero mode associated with translational symmetry,

$$
Z_{0}(\tau)=\sqrt{\frac{3 m}{8}} \frac{1}{\cosh ^{2}\left[\frac{m}{2}(\tau-X)\right]},
$$

where we have introduced the variable $m=\mu \sqrt{2}$. This zero mode is obtained by differentiation of the kink-instanton with respect to the collective coordinate. The normalization is chosen such that $\int \mathrm{d} \tau Z_{0}(\tau) Z_{0}(\tau)=1$. Evaluating the action on the kink-instanton yields

$$
S_{\text {inst }}^{(0)}=-\frac{2 \sqrt{2} \mu^{3}}{3 \lambda}=-\frac{m^{3}}{3 \lambda} .
$$

Tunnelling phenomena, including two-loop effects, in this model were analyzed in [9].

We are interested in models with fermions, so we add the following terms

$$
S_{2-\text { ferm }}=-\frac{1}{2} \int \mathrm{d} \tau\left\{\psi_{i}^{T} \dot{\psi}_{i}+\left(\psi_{i}^{T} \sigma_{2} \psi_{i}\right) U^{\prime}\right\}
$$


Here $i$ is a flavor index, and the fermions are two-component spinors. For one fermion, there is a supersymmetry, given by

$$
\delta x=\varepsilon^{T} \sigma^{2} \psi ; \quad \delta \psi=\sigma^{2} \dot{x} \varepsilon-U \varepsilon,
$$

but we are interested in the case $i=1, \ldots, 4$. The field equations read

$$
\begin{aligned}
\ddot{x}-U U^{\prime} & =\frac{1}{2}\left(\psi_{i}^{T} \sigma^{2} \psi_{i}\right) U^{\prime \prime} \\
\dot{\psi}_{i}+\sigma^{2} \psi_{i} U^{\prime} & =0 .
\end{aligned}
$$

The usual instanton solution to this set of equations is obtained by setting all fermions to zero and taking for $x$ the kink-instanton, $x_{K}$. In the supersymmetric case this solution is invariant under SUSY transformations with parameter $\varepsilon=\frac{1-\sigma^{2}}{2} \varepsilon$, while the parameter $\varepsilon=\frac{1+\sigma^{2}}{2} \varepsilon$ breaks SUSY and generates a fermionic zero mode.

There is another solution, for which the fermions are not zero. It contains $x=x_{K}(\tau)$ and the fermion zero modes

$$
\psi_{i}^{(1)}(\tau)=\xi_{i} Z_{0}(\tau) \psi_{+},
$$

where we have introduced the spinor

$$
\psi_{+}=\frac{1}{\sqrt{2}}\left(\begin{array}{l}
1 \\
i
\end{array}\right) .
$$

The $\xi_{i}$ are Grasmannian collective coordinates and $Z_{0}(\tau)$ is the bosonic zero mode in (2.4).

The fermionic field equation is satisfied because $\sigma^{2} \psi_{+}=\psi_{+}$and $\dot{Z}_{0} \propto \ddot{x}_{K}$ while $\dot{x}_{K}+U\left(x_{K}\right)=0$ according to the BPS equation. For the bosonic field equation one notices that the inhomogeneous term on the right hand side is zero for two reasons: the product $\xi_{i} \xi_{i}$ vanishes but also $\psi_{+}^{T} \sigma^{2} \psi_{+}=0$. The instanton action for this solution is still given by (2.5), because the fermion terms vanish upon using the field equations. For $i=1$ the solution in (2.9) is generated by using the broken SUSY in $\delta \psi$ from (2.7). The normalization is chosen such that the zero modes

$$
Z_{[i]}^{j} \equiv \frac{\partial \psi_{i}^{(1)}}{\partial \xi_{j}},
$$

are orthonormal,

$$
\left\langle Z^{i} \mid Z^{j}\right\rangle \equiv \sum_{k} \int \mathrm{d} \tau\left(Z_{[k]}^{i}\right)_{\alpha}^{*}\left(Z_{[k]}^{j}\right)^{\alpha}=\delta^{i j}
$$

To make the model more interesting, we deform it by adding a four-fermi term to the action,

$$
S_{4-\mathrm{fermi}}=\frac{g}{4} \int \mathrm{d} \tau \epsilon^{i j k l}\left(\psi_{i}^{T} \sigma^{1} \psi_{j}\right)\left(\psi_{k}^{T} \sigma^{1} \psi_{l}\right)
$$

where $g$ is a dimensionful coupling constant. There are actually two terms which are Lorentz invariant in Minkowski spacetime: a product of pseudo-scalars (with a $\sigma^{3}$ inserted), or an inner 
product of vector terms, with $\sigma^{\mu}$ inserted. One can eliminate the vector terms in favor of the pseudo-scalar term.

The field equations now take the form

$$
\begin{aligned}
\ddot{x}-U U^{\prime} & =\frac{1}{2}\left(\psi_{i}^{T} \sigma^{2} \psi_{i}\right) U^{\prime \prime} ; \quad U^{\prime}=m \tanh \left[\frac{m}{2}(\tau-X)\right], \quad U^{\prime \prime}=\sqrt{2 \lambda} \\
\dot{\psi}_{i}+\sigma^{2} \psi_{i} U^{\prime} & =g \epsilon_{i j k l} \sigma^{1} \psi_{j}\left(\psi_{k}^{T} \sigma^{1} \psi_{l}\right) .
\end{aligned}
$$

We want to find an exact solution to these equations by iterating in the number of GCC. Substituting the fermionic zero mode solution into the right hand side of the fermionic field equation, one determines the part of $\psi_{i}$ which is cubic in the GCC,

$$
\dot{\psi}_{i}^{(3)}+\sigma^{2} \psi_{i}^{(3)} U^{\prime}=-g\left(\frac{3 m}{8}\right)^{3 / 2}\left(\epsilon_{i j k l} \xi_{j} \xi_{k} \xi_{l}\right) \frac{1}{\cosh ^{6}\left[\frac{m}{2}(\tau-X)\right]} \psi_{-} .
$$

We used $\psi_{+} \sigma^{1} \psi_{+}=2 i$ and introduced the spinor $\psi_{-}=-i \sigma^{1} \psi_{+}$,

$$
\psi_{-}=\frac{1}{\sqrt{2}}\left(\begin{array}{c}
1 \\
-i
\end{array}\right)
$$

which is annihilated by $\left(1+\sigma^{2}\right)$.

Making the ansatz

$$
\psi_{i}^{(3)}=\alpha(\tau)\left(\epsilon_{i j k l} \xi_{j} \xi_{k} \xi_{l}\right) \psi_{-},
$$

$\alpha$ is determined by

$$
\dot{\alpha}-\alpha m \tanh \left[\frac{m}{2}(\tau-X)\right]=-g\left(\frac{3 m}{8}\right)^{3 / 2} \frac{1}{\cosh ^{6}\left[\frac{m}{2}(\tau-X)\right]} .
$$

Since $\cosh ^{2}\left[\frac{m}{2}(\tau-X)\right]$ is a solution of the homogeneous equation, we set

$$
\alpha(\tau)=\cosh ^{2}\left[\frac{m}{2}(\tau-X)\right] y(\tau)
$$

The differential equation then reduces to

$$
\dot{y}=-g\left(\frac{3 m}{8}\right)^{3 / 2} \frac{1}{\cosh ^{8}\left[\frac{m}{2}(\tau-X)\right]} .
$$

Changing variables to $z=\tanh \left[\frac{m}{2}(\tau-X)\right]$, we obtain, with $c=-g \frac{2}{m}\left(\frac{3 m}{8}\right)^{3 / 2}$,

$$
\frac{\mathrm{d} y}{\mathrm{~d} z}=c\left(1-z^{2}\right)^{3}
$$

which is solved by

$$
y=c\left(a+z-z^{3}+\frac{3}{5} z^{5}-\frac{1}{7} z^{7}\right)
$$


where $a$ is an integration constant.

Looking at the behaviour at $\tau= \pm \infty$, which corresponds to $z= \pm 1$, we see that $\alpha(\tau= \pm \infty)$ diverges for generic values of $a$. Even if we choose $a$ such that $y(z)$ vanishes at $z=-1$ (namely $\left.a=\frac{3}{5}-\frac{1}{7}=\frac{16}{35}\right), \alpha(\tau)$ still diverges at $\tau=\infty$,

$$
\alpha(\tau \rightarrow+\infty) \rightarrow \frac{8 c}{35} \mathrm{e}^{m \tau}
$$

The solution for $\psi_{i}^{(3)}$ in the fermionic background now acts as a source term for the bosonic field equation. Denoting the new term in $x$ proportional to $\epsilon \xi^{4} \equiv \epsilon^{i j k l} \xi_{i} \xi_{j} \xi_{k} \xi_{l}$ by $x^{(4)}$, one must solve $x^{(4)}$ from the equation of motion for $x=x_{K}+x^{(4)}$,

$$
\ddot{x}^{(4)}-x^{(4)}\left(U U^{\prime \prime}+U^{\prime} U^{\prime}\right)\left(x_{K}\right)=\left(\psi_{i}^{(1) T} \sigma^{2} \psi_{i}^{(3)}\right) U^{\prime \prime}
$$

Substituting (2.9), (2.17) and (2.19) leads to

$$
\ddot{x}^{(4)}-\frac{m^{2}}{2} x^{(4)}\left(3 \tanh ^{2}\left[\frac{m}{2}(\tau-X)\right]-1\right)=-\sqrt{2 \lambda} \sqrt{\frac{3 m}{8}}\left(\epsilon \xi^{4}\right) y(\tau),
$$

with $y(\tau)$ given in (2.22).

The homogeneous equation is of course solved by the bosonic zero mode $Z_{0}(\tau)$, so we make the ansatz

$$
x^{(4)}(\tau)=\frac{\beta(\tau)}{\cosh ^{2}\left[\frac{m}{2}(\tau-X)\right]} .
$$

In the same $z$ variable as before, we find the following differential equation for $\beta$,

$$
\left(1-z^{2}\right) \frac{\mathrm{d}^{2} \beta}{\mathrm{d} z^{2}}-6 z \frac{\mathrm{d} \beta}{\mathrm{d} z}=\frac{1}{\left(1-z^{2}\right)^{2}} \sqrt{2 \lambda}\left(\epsilon \xi^{4}\right) g \frac{9}{8 m}\left(a+z-z^{3}+\frac{3}{5} z^{5}-\frac{1}{7} z^{7}\right)
$$

The homogeneous equation is solved by $\mathrm{d} \beta / \mathrm{d} z=A /\left(1-z^{2}\right)^{3}$, with $A$ an integration constant, which, as we mentioned above, we take proportional to $\epsilon \xi^{4}$. So we write $A\left(\epsilon \xi^{4}\right)$ instead. The general solution is then written as

$$
\frac{\mathrm{d} \beta}{\mathrm{d} z}=\frac{A\left(\epsilon \xi^{4}\right)}{\left(1-z^{2}\right)^{3}}+\frac{\gamma(z)}{\left(1-z^{2}\right)^{3}} .
$$

This yields a first order differential equation for $\gamma$,

$$
\frac{\mathrm{d} \gamma}{\mathrm{d} z}=\sqrt{2 \lambda}\left(\epsilon \xi^{4}\right) g \frac{9}{8 m}\left(a+z-z^{3}+\frac{3}{5} z^{5}-\frac{1}{7} z^{7}\right)
$$

which can easily be integrated to give

$$
\frac{\mathrm{d} \beta}{\mathrm{d} z}=\frac{\left(\epsilon \xi^{4}\right)}{\left(1-z^{2}\right)^{3}}\left[A+\sqrt{2 \lambda} g \frac{9}{8 m}\left(a z+\frac{1}{2} z^{2}-\frac{1}{4} z^{4}+\frac{1}{10} z^{6}-\frac{1}{56} z^{8}\right)\right] .
$$


One can integrate again,

$$
\begin{aligned}
\beta(z)= & \left(\epsilon \xi^{4}\right)\left(A-\sqrt{2 \lambda} g \frac{9}{64 m}\right) \frac{3}{16} \ln \frac{1+z}{1-z}+\frac{A\left(\epsilon \xi^{4}\right)}{8} \frac{5 z-3 z^{3}}{\left(1-z^{2}\right)^{2}} \\
& +\sqrt{2 \lambda}\left(\epsilon \xi^{4}\right) g \frac{9}{8 m}\left[B+\frac{1}{\left(1-z^{2}\right)^{2}}\left(\frac{a}{4}+\frac{1}{24}\left(\frac{9}{8} z+\frac{17}{8} z^{3}-\frac{7}{5} z^{5}+\frac{1}{7} z^{7}\right)\right)\right],
\end{aligned}
$$

where $B$ is another integration constant.

It is clear that also $x^{(4)}$ diverges in general at $\tau= \pm \infty(z= \pm 1)$, but by choosing $A$ and $a$ appropriately, $x^{(4)}$ is bounded at infinity, see the discussion in the next section.

Notice that we have now obtained an exact solution to the full equations of motion. Indeed, the iteration in GCC stops at fourth order in the $\xi_{i} ; i=1, \ldots, 4$, so there is no further contribution to either $\psi_{i}$ or $x$. The solution is given by

$$
x=x_{K}+x^{(4)}, \quad \psi_{i}=\psi_{i}^{(1)}+\psi_{i}^{(3)},
$$

with $x^{(4)}$ given in (2.26) and (2.31), $\psi_{i}^{(1)}$ given in (2.9) and $\psi_{i}^{(3)}$ in (2.17), (2.19) and (2.22).

We now compute the instanton action. First of all, the four-fermi term gives a contribution

$$
S_{4-\text { fermi }}=-g \frac{9 m}{128}\left(\epsilon \xi^{4}\right) \int_{-1}^{1}\left(1-z^{2}\right)^{3} \mathrm{~d} z=-g \frac{9 m}{140}\left(\epsilon \xi^{4}\right)
$$

Next we evaluate the two-fermi terms. Using the field equation for $\psi_{i}^{(1)}+\psi_{i}^{(3)}$, we find that their contribution to the action has opposite sign and is twice as large as the one from the four-fermi term,

$$
S_{2-\text { fermi }}=g \frac{9 m}{70}\left(\epsilon \xi^{4}\right) .
$$

Finally, we consider the bosonic sector, for which the relevant contribution to the action can be written as

$$
S_{\mathrm{bos}}=-\int \mathrm{d} \tau\left[\dot{x}_{K} \dot{x}^{(4)}+x^{(4)} U^{\prime}\left(x_{K}\right) U\left(x_{K}\right)\right] .
$$

Partially integrating and using the field equations for $x_{K}$ we obtain

$$
S_{\mathrm{bos}}=-\int \mathrm{d} \tau \frac{\mathrm{d}}{\mathrm{d} \tau}\left(\dot{x}_{K} x^{(4)}\right)=-\frac{m^{2}}{2 \sqrt{2 \lambda}}\left[\left(1-z^{2}\right)^{2} \beta(z)\right]_{-1}^{1},
$$

where $\beta(z)$ is as in (2.31). This is precisely the surface term mentioned in the introduction. So we find

$$
S_{\mathrm{bos}}=-\left(\epsilon \xi^{4}\right)\left(\frac{m^{2} A}{4 \sqrt{2 \lambda}}+g \frac{9 m}{16} \frac{93}{560}\right) .
$$

It is clear that $S_{\text {bos }}$ depends on the collective coordinate $A$ but not on $a$ and $B$. The term in $x$ proportional to $A$ is the zero-frequency fluctuation which is not normalizable . It blows up at $\tau= \pm \infty$, but it contributes a finite amount to the action because $\dot{x}_{K}$ vanishes rapidly at $\tau= \pm \infty$.

\footnotetext{
${ }^{4}$ The other zero-frequency solution is $Z_{0}$ which is, of course, normalizable.
} 
Collecting the bosonic and fermionic contributions from (2.5), (2.37), (2.34) and (2.33), we obtain the final result for the instanton action

$$
S=-\frac{m^{3}}{3 \lambda}-\left(\frac{m^{2} A}{4 \sqrt{2 \lambda}}+g \frac{261 m}{8960}\right) \epsilon^{i j k l} \xi_{i} \xi_{j} \xi_{k} \xi_{l} .
$$

Hence although $x$ and $\psi_{i}$ blow up at $\tau= \pm \infty$, they are regular at all finite $\tau$ and have finite action.

We are currently studying the relation between this solution and the original, purely bosonic one. It may be that one can obtain (2.38) by computing the finite number of tree graphs with four fermionic zero modes on the external legs. The propagator for scalars in the background of $x_{K}$ has been worked out in [9] and for fermions in [10].

\section{Discussion}

We have obtained a new solution of the Euclidean coupled field equations of the double well potential in quantum mechanics with a particular four-fermion interaction. The solution depends on four fermionic collective coordinates $\xi_{i} ; i=1, \ldots, 4$ and contains further three integration constants $A, B, a$, but the action is independent of $B$ and $a$. By requiring that the bosonic field $x(\tau)$ remains bounded as $\tau \rightarrow \pm \infty$, we find that $a=0$ but $A$ gets fixed to $A=-\sqrt{2 \lambda} \frac{g}{m} \frac{9 \cdot 93}{16 \cdot 140}$. For this value of $A$, the contribution (2.37) from the boson vanishes and the instanton action becomes

$$
S=-\frac{m^{3}}{3 \lambda}+\frac{9}{140} g m \epsilon^{i j k l} \xi_{i} \xi_{j} \xi_{k} \xi_{l} .
$$

We have called the result of this paper a new instanton, but is our solution an instanton ? Instantons are usually defined as solutions of the Euclidean equations of motion with finite action. However, one can also define them as configurations describing tunnelling processes between different vacua in Minkowski space. The usual instanton solutions [1, 2] satisfy both definitions. Our solution clearly satisfies the first definition. Concerning tunnelling, the interpretation of our solution is less clear. There are vacua with non-vanishing values for the fermions, but they are of a different nature from the usual vacua. Our solution with $a=0$ and $A$ given above, interpolates between two configurations with the same energy, but this energy is non-vanishing and proportional to $\epsilon^{i j k l} \xi_{i} \xi_{j} \xi_{k} \xi_{l}$. We intend to study the interpretation of our solution and whether it describes tunnelling between two such vacua.

Is our solution new ? We have had detailed discussions with A. Vainshtein concerning the relation between our construction and the work of V.A. Novikov, M.A. Shifman, A.I. Vainshtein, and V.I. Zakharov (NSVZ) on supersymmmetry transformations of collective coordinates [3]. Our approach is dynamical; it uses field equations and holds for both non-supersymmetric and supersymmetric models. Moreover, we do not use spontaneous symmetry breaking and constrained 
instantons at intermediate levels. On the other hand, the work of NSVZ starts from symmetry considerations, in particular supersymmetry and superconformal supersymmetry. Dynamics enters only in sofar as spontaneous symmetry breaking produces a vacuum expectation value $v$. The supersymmetry transformations act between both the (bosonic and fermionic) collective coordinates and $v$. Although constrained instantons are needed in this approach with $v \neq 0$, one can afterwards take the limit $v \rightarrow 0$ and regain results which should correspond to the results for unbroken $(v=0) \mathcal{N}=4$ supersymmetric gauge theories [6, ], as was demonstrated in [8]. We intend to work out the relation between both approaches in the future. In the work of NSVZ, supersymmetric gauge theories with $S U(2)$ were considered. The case of higher $S U(N)$ was treated in [12].

\section{References}

[1] A. Belavin, A. Polyakov, A. Schwartz and Y. Tyupkin, Phys. Lett. B 59 (1975) 85.

G. 't Hooft, Phys. Rev. D 14 (1976) 3432.

[2] E. Gildener and A. Patrascioiu, Phys. Rev. D 16 (1977) 423.

R. Rajaraman, Solitons and Instantons, North-Holland, (Amsterdam, 1982).

S. Coleman, The uses of instantons, in Proc. Int. School of Subnuclear Physics, Erice (1977), and in Aspects of Symmetry, Cambridge University Press, (Cambridge, 1985) 265.

A. Vainshtein, V. Zakharov, V. Novikov and M. Shifman, ABC of Instantons, Sov. Phys. Usp. 25 (1982) 195, and in Instantons in Gauge Theories, ed. M. Shifman, World Scientific, (Singapore, 1994).

[3] V. A. Novikov, M. A. Shifman, A. I. Vainshtein and V. I. Zakharov, Nucl. Phys. B 229 (1983) 394; Nucl. Phys. B 229 (1983) 407; Nucl. Phys. B 260 (1985) 157; Phys. Lett. B 217 (1989) 103.

[4] G.H. Derrick, J. Math. Phys. 5 (1964) 1252;

R. Hobart, Proc. Royal. Soc. London 82 (1963) 201.

[5] I. Affleck, Nucl. Phys. B 191 (1981) 429. For a recent analysis, see M. Nielsen and N.K. Nielsen, Phys. Rev. D 61: 105020, 2000, hep-th/9912006.

[6] N. Dorey, V.V. Khoze, M.P. Mattis and S. Vandoren, Phys. Lett. B 442 (1998) 145, hepth/9808157.

\footnotetext{
${ }^{5}$ The limit $v \rightarrow 0$ must be taken with care, and might not always be the same as starting with $v=0$ [11].
} 
N. Dorey, T. Hollowood, V.V. Khoze, M.P. Mattis and S. Vandoren, Nucl. Phys. B 552 (1999) 88, hep-th/9901128.

[7] A. Belitsky, S. Vandoren and P. van Nieuwenhuizen, Class. Quantum Grav. 17 (2000) 3521, hep-th/0004186; Phys. Lett. B 477 (2000) 335, hep-th/0001010.

[8] N. Dorey, V.V. Khoze and M.P. Mattis, Phys. Lett. B 396 (1997) 141, hep-th/9612231.

[9] A.A. Aleinikov and E.V. Shuryak, Sov. J. Nucl. Phys. 46 (1987) 76;

S. Olejnik, Phys. Lett. B 221 (1989) 372;

C.F. Wöhler and E.V. Shuryak Phys. Lett. B 333 (1994) 467, hep-ph/9402287.

[10] P. van Nieuwenhuizen, Instantons and tunnelling in quantum mechanics, Stony Brook preprint, YITP-SB-00-62.

[11] J. Fuchs and M. G. Schmidt, Z. Phys. C 30 (1986) 161.

[12] J. Fuchs, Nucl. Phys. B 272 (1986) 677; Nucl. Phys. B 282 (1987) 437.

V. A. Novikov, Sov. J. Nucl. Phys. 46 (1987) 554, also in Instantons in Gauge Theories, ed. M. Shifman, World Scientific, (Singapore, 1994). 